Review

\title{
Signal Transducers and Activators of Transcription (STAT) Family Members in Helminth Infections
}

\author{
Mireya Becerra-Díaz ${ }^{1}$, Héctor Valderrama-Carvajal ${ }^{2}$ and Luis I. Terrazas ${ }^{1 凶}$ \\ 1. Unidad de Biomedicina, Facultad de Estudios Superiores-Iztacala, Universidad Nacional Autónoma de México (UNAM). \\ 2. Instituto de Ciencia y Tecnología, Distrito Federal, México.
}

$\triangle$ Corresponding author: Luis I. Terrazas, Unidad de Biomedicina. FES-Iztacala, UNAM. Av. De los Barrios 1, Los Reyes Iztacala, Tlalnepantla, Edo. de México. México 54090. Phone: (5255) 5623-1333 ext 39794. Fax: (5255) 5623-1138. e-mail: literrazas@campus.iztacala.unam.mx

(C) Ivyspring International Publisher. This is an open-access article distributed under the terms of the Creative Commons License (http://creativecommons.org/ licenses/by-nc-nd/3.0/). Reproduction is permitted for personal, noncommercial use, provided that the article is in whole, unmodified, and properly cited.

Received: 2011.09.01; Accepted: 2011.10.01; Published: 2011.11.01

\begin{abstract}
Helminth parasites are a diverse group of multicellular organisms. Despite their heterogeneity, helminths share many common characteristics, such as the modulation of the immune system of their hosts towards a permissive state that favors their development. They induce strong Th2-like responses with high levels of IL-4, IL-5 and IL-I 3 cytokines, and decreased production of proinflammatory cytokines such as IFN- $\gamma$. IL-4, IFN- $\gamma$ and other cytokines bind with their specific cytokine receptors to trigger an immediate signaling pathway in which different tyrosine kinases (e.g. Janus kinases) are involved. Furthermore, a seven-member family of transcription factors named Signal Transducers and Activators of Transcription (STAT) that initiate the transcriptional activation of different genes are also involved and regulate downstream the JAK/STAT signaling pathway. However, how helminths avoid and modulate immune responses remains unclear; moreover, information concerning STAT-mediated immune regulation during helminth infections is scarce. Here, we review the research on mice deficient in STAT molecules, highlighting the importance of the JAK/STAT signaling pathway in regulating susceptibility and/or resistance in these infections.
\end{abstract}

Key words: STAT, helminths, Taenia crassiceps, alternatively activated macrophages, filariasis, schistosomiasis.

\section{Introduction}

Helminth parasites are a very diverse group of multicellular organisms, including both flat worms like Taenia sp., and cylindrical worms like Ascaris sp. Although they exhibit different mechanisms of infection and distinct sites at which they lodge in the host, they share some common features such as the immune modulation induced by the host immune system that triggers a permissive state for their development [1]. Helminth parasites possess sophisticated and efficient mechanisms to regulate the immune response to survive inside their hosts. A distinctive characteristic in these infections is that they induce the skewing of the immune response toward a Th2 re- sponse with high concentrations of interleukin (IL)-4, IL-13, IL-5 and IgE, IgG1 and IgG4 isotype antibodies that commonly are related to protective effects [2]. On the other hand, a hypo-responsive state is also consistently observed in lymphocytes, mainly by a reduced proliferative response to parasites, non-related antigens, or polyclonal stimuli [3]. In addition to Th2 polarization, the recruitment of immunomodulatory cell populations, such as $T$ regulatory cells (Tregs) and alternatively activated macrophages (AAMФs), has also been recently described in different helminthic infections, and these cells may be dependent on such signals. The role of this group of cells depends on the 
particular type of helminth. For example, in Nippostrogylus brasiliensis infection, ААМФs actively participate in parasite clearance, while in Taenia crassiceps infection; $\mathrm{AAM} \Phi$ s favor parasite persistence $[4,5]$. Interestingly, experimental infections with Brugia malayi, Schistosoma mansoni or Litomosoides sigmodontis induce an increased and rapid recruitment of $\mathrm{CD}^{+}{ }^{+} \mathrm{CD} 25^{+}{ }^{+}$oxp $^{+}{ }^{+}$Tregs $[6,7,8]$, which can be beneficial for both, the parasite and the host. It is clear that the strong regulatory mechanisms developed by helminths are necessary either to successfully colonize their hosts or to complete their life cycle while minimizing damage to the host. However, the mechanisms used by helminth parasites to achieve modulation of the host immune response have not been clarified.

During helminth infection, high levels of different types of cytokines are secreted. Cytokines initiate signaling when they bind to their specific receptors, inducing several important conformational changes mainly oligomerization or multimerization of their receptors. These changes are followed by activation of several downstream signaling molecules including those named JAK (Janus Kinases). JAKs signaling molecules are tyrosine kinases, found constitutively associated with each receptor chain [9]. Once the JAKs are activated, they autophosphorylate and transphosphorylate the receptor tyrosine intracellular motifs, which serve as recruiting sites for the SH2 domain of STATs.

Upon activation, the STATs are phosphorylated and dimerize through their $\mathrm{SH} 2$ domain to form homo- or heterodimers. These dimers translocate to the nucleus where they bind to the promoter region of genes via specific DNA binding domains and thus bring about the transcription of their respective genes. Here, we discuss the signature cytokines and complex STAT-mediated signaling networks involved in regulating the host response and determining disease outcome during various helminth infections.

\section{The JAK/STAT families}

JAK/STAT families are two groups of proteins that, constitute diverse signaling pathways [10] involved in cytokine signaling. STAT family members act as transcriptional factors, activated upstream by JAK proteins. Furthermore, STAT proteins are phosphorylated on tyrosine residues by JAKs. The STAT family is composed of seven proteins (STAT1, STAT2, STAT3, STAT4, STAT5a, STAT5b and STAT6), while the JAK family is composed of four proteins (JAK1, JAK2, JAK3 and TyK2). All of these proteins are constitutively present in the cytoplasm without previous stimuli. These two large groups of molecules repre- sent a signaling pathway that can be quickly activated from the cellular membrane to the nucleus.

\section{JAK protein family}

These proteins are called Janus kinases because of the homology of the kinase and pseudokinase domains with Janus, the Roman god of two faces [11]. The first JAK identified was Tyk2 by Krolewski in 1990 [12], using libraries of complementary DNA from human $\mathrm{T}$ lymphocytes, while JAK1, JAK2 and JAK3 where identified through conserved motif clonation of the catalytic domain [11, 13, 14]. JAK proteins are molecules structurally composed of seven regions (JH1- JH7). The JH1 domain bears the kinase activity (Fig.1), while JH2 is homologous to JH1 but without the residues required for kinase activity (pseudokinase domain), so this domain is usually associated with regulatory functions [15].

JAK proteins interact with different intracellular domains of cytokine receptors and are present in a variety of cell subtypes. Their participation in mutated cell lines resistant to IFN- $\alpha$ stimuli was observed, and signaling in those cells was restored when different JAK proteins were transfected. Thus it was shown that IFN- $\alpha$ signaling requires JAK1 and Tyk2, while IFN- $\gamma$ signaling requires JAK1 and JAK2 [16. 17, 18]. After that discovery, the participation of other JAK proteins in cytokine signaling was described (Table 1).

As shown in Table 1, although JAKs participate in multiple signaling pathways, their importance in modulating immune responses is evident. Therefore, their function is mainly in cytokine signaling.

Table I. JAK Family

\begin{tabular}{ll}
\hline & Cytokine or factor \\
\hline JAK1 & IL-2, IL-7, IL-9, IL-15, IL-4, IL-13, IL-6, IL-11, \\
& IFN- $\alpha$, IFN- $\beta$, IFN- $\gamma$, IL-10, CT-1 \\
JAK2 & IL-3, IL-12, IL-13, IL-6, IL-11, IFN- $\gamma$, CT-1, \\
& Growth hormone, Prolactin, Eritropoyetin \\
JAK3 & IL-2, IL-7, IL-9, IL-15, IL-4 \\
TyK2 & IL-6, IL-11, IL-12, IL-13, CT-1, IFN- $\alpha$, IFN- $\beta$, \\
& IL-10 \\
\hline
\end{tabular}

\section{STAT family}

The STAT proteins are a family of transcription factors composed of 7 members. In 1994, Darnell and colleagues identified the first two members of the family, STAT1 and 2, by purification of factors linked to IFN stimulated genes; the other family members were described subsequently and are STAT3, STAT4, STAT5a, STAT5b and STAT6 [19, 20, 21, 22, 23]. These 
proteins act as transcription factors when they form homo- and heterodimers among them. Dimerization is possible once STATs have been phosphorylated at tyrosine residues in their SH2 domain (Fig.1). STATs are the only family of transcription factors that contain $\mathrm{SH} 2$ domains for phosphotyrosines that serve mainly for both binding to the activated cytokine receptor and activating STATs through tyrosine phosphorylation [24]. STAT proteins together with JAKs are involved in many cytokine signaling pathways (Table 2).

Table 2. STAT Family

\begin{tabular}{ll}
\hline & Cytokine or factor \\
\hline STAT1 & IL-2, IL-6, IL-10, IFN- $\alpha$, IFN- $\beta$, IFN- $\gamma$, IL-27 \\
STAT2 & IFN- $\alpha$, IFN- $\beta$ \\
STAT3 & LIF, IL-10, IL-6, IL-27, Growth hormone \\
STAT4 & IL-12 \\
STAT5a/b & Prolactin, Growth hormone, Thrombopoietin \\
STAT6 & IL-4, IL-13 \\
\hline
\end{tabular}

The Janus kinase/signal transducers and activators for transcription (JAK/STAT) pathway regulate a large plethora of biological processes including cell proliferation, differentiation, cell migration and apoptosis [25]. Ligand binding induces an intracellular activation through the multidimerization of different cytokine receptors units [25], followed by JAKs recruitment to the cytoplasmatic domain of the cytokine receptor. JAK activation occurs when a tyrosine residue in the cytokine receptor is phosphorylated, creating a docking site for cytoplasmic STAT. While STAT proteins are attached to the cytokine receptor, JAK proteins phosphorylate them at a tyrosine residue, detaching the STAT protein from the cytokine receptor so that the STATs form homo- and heterodimers that will translocate to the nucleus and bind DNA sequences to promote gene expression (Fig. 2).

An important characteristic observed in diverse helminthic infections is the participation of some members of the STAT protein family and their associated tyrosine kinases family (JAK kinases) in the regulation of susceptibility and resistance to the infection. Several reports have shown that STAT proteins participate in diverse cytokines signaling pathways, such as IL-4 and IL-13, particularly involving STAT6, responsible for triggering the Th2 immune responses at the transcriptional level, which is a feature of helminth infections.

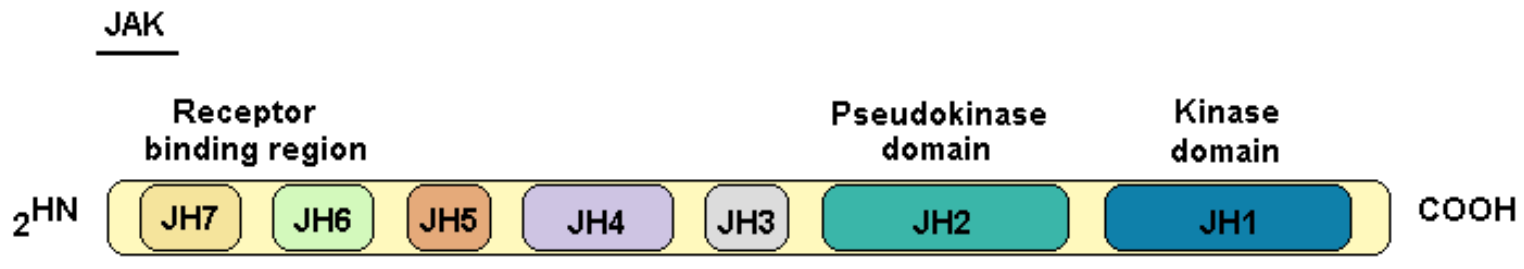

STAT

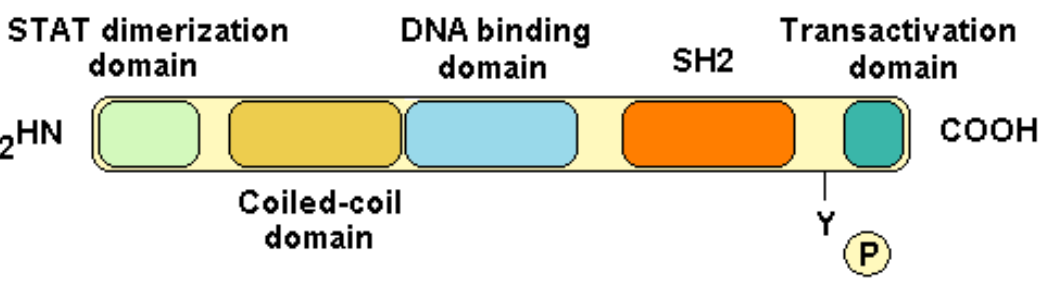

Figure I. JAK and STAT protein structures. JAK proteins feature 7 domains; they owe their name due to the homology of $\mathrm{JH}$ and $\mathrm{JH} 2$ to the two faced god Janus. The difference between both domains is that $\mathrm{JH} 2$ lacks some residues implicated in kinase activity. STAT proteins are transcription factors that feature five conserved domains. 
Figure 2. JAK/STAT signaling pathway. Cytokine binds with the target receptor; this causes dimerization of receptor chains and the juxtaposition of JAKs, and their reciprocal phosphorylation. Once activated, JAKs phosphorylate the intra-cytoplasmic regions of the cytokine receptor; creating a docking site for cytoplasmatic STATs that once joined with the receptor are activated by JAK phosphorylation in a tyrosine residue. STATs dimers act as transcriptional factors recognizing specific DNA sequences.

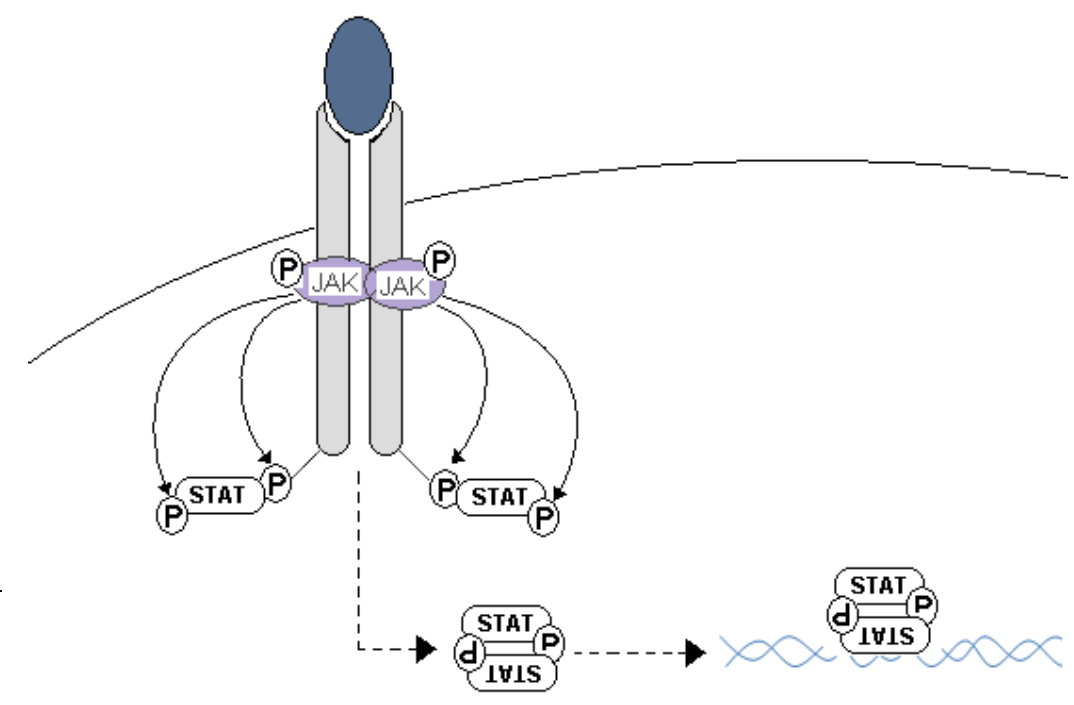

IFN- $\gamma$ is a cytokine affected by helminth infections. One of the most important functions of this cytokine is classic activation of macrophages, and it is also known that chronic infections with this kind of parasite preferentially induce an alternative state of activation in this cell lineage. It has been shown that ААМФs are induced by stimulation with IL-4, and they do not have the faculty to adequately respond to IFN- $\gamma[26,27]$. This may be the key to the relationship between helminth infections and the JAK/STAT proteins because is STAT1 precisely the transcription factor involved in the IFN- $\gamma$ signaling pathway.

\section{JAK/STAT in helminth infections}

Large amount of information has demonstrated that the JAK/STAT pathway is involved in helminth infections, suggesting that family members of these families might participate in mediating susceptibility or resistance to different helminth infections.

Helminth parasites are a heterogeneous group with common characteristics, such as host modulation of the immune response to generate a permissive environment that favors infection and induction of the development and polarization of the immune response towards a Th2 dominant profile. It is known that development of Th2 immune responses is IL-4-dependent, while the Th1 immune responses depend mainly on IL-12 and IFN- $\gamma$ [28]. These important cytokines exert their biological effects signaling through the JAK/STAT pathway. However, the mechanisms involved in helminth immune modulation remain unclear, but there is evidence that indicates the importance of the presence or absence of some JAK/STAT proteins to modify the resistance or susceptibility to these parasite infections

\section{STATI and helminths}

STAT1 is an important player in IFN- $\gamma$ signaling. It has been demonstrated that IFN- $\gamma$ is a very important cytokine in the development and regulation of Th1-type immune response and inflammatory responses. Whereas IFN- $\gamma$ plays a critical role in protecting animals against viral, bacterial, and protozoan pathogens, its role on many helminthic infections is practically unknown. Moreover, it was previously thought that Th1-mediated immune responses did not participate as effector responses against helminth infections. However, several new evidences show that immune response against these parasites is a very complex event. Effectors' mechanisms against helminths are not exclusively limited to Th2-type responses, but Th1-type responses may participate and develop effector mechanisms depending on diverse factors such as the type of helminth infection, site where the helminth is established (intestinal lumen, liver, muscle, lung or brain), and the life cycle stage of the parasite (eggs, larvae or adult).

In contrast to other STAT family proteins, very few reports exist regarding how STAT1 participates in helminth infections. Schistosomiasis is a parasitic disease caused by trematode flatworms of the genus Schistosoma, and in particular Schistosoma mansoni, affecting both human and cattle. The immune response against this parasite is initiated by soluble egg antigens (SEA) derived by laying eggs in the lung and liver. During the development of the infection, a significant decrease of Th1-type cytokine (IL-12, IFN- $\gamma$ and TNF- $\alpha$ ) expression has been observed followed by a clear augmentation in the production of regulatory and Th2-type cytokine (TGF- $\beta$, IL-10, IL-4) ex- 
pression in splenocytes, protecting host-pathology and control etiology of sickness [29].

Due to the fact that STAT1 is not only involved in the Th1 response but also in regulating cytokine signaling (i.e., IL-10), Goh and colleagues [30] analyzed the effect of SEA in activating different signaling pathways that could induce Th2 immune responses. Interestingly, among the molecules activated by SEA, this group observed that p38 and Erk 1/2 signaling pathways were activated but STAT1 was not.

In murine schistosomiasis, IFN- $\gamma$ and the Th1 response can protect against severe fibrosis by preventing $А A M \Phi$ activation and thereby limiting the fibrosis by enhancing effects of the Th2 response [31]. However, in humans Mwatha and colleagues [32] reported Th1-type immune responses in individuals with severe hepatosplenomegaly, whereas individuals infected but with less-severe hepatosplenomegaly mounted Th2-type responses. Thus, STATs controlling Th1 or Th2 responses may be involved in regulating the balance between pathology and elimination of this helminth infection.

In contrast to the dearth of knowledge regarding participation of STAT1 in helminth infections, there is evidence of its importance in protozoan infections. When C57BL/ 6 mice that mount a strong Th1 response against Leishmania major infection lack STAT1, they became highly susceptible to this infection [33]. In some helminth infections such as filariasis, cysticercosis and echinococcosis, the early immune response is Th1-mediated, which sometimes correlates with rapid control of the infection or maintenance of low parasite burdens. However, this response is mainly transient, and it rapidly shifts towards Th2 immune responses that may favor the establishment of the helminth. Keeping in mind that STAT1 is key molecule in Th1-type immune response development, this suggests that a rigorous study on STAT1 participation in helminth infections is clearly missing in the literature of the immunoparasitology field, therefore we cannot rule out a possible role for STAT1 in regulating control or pathology of helminth infections.

\section{A role for STAT4 during helminth infections}

The transcription factor STAT4 participates in the IL-12 signaling pathway. IL-12 functions as the main physiological inducer of IFN- $\gamma$ by activated $T$ cells and promotes Th1-type $\mathrm{CD}^{+} \mathrm{T}$ cell differentiation and therefore may play an important role in response to helminth infections. Thus, STAT4 and STAT1 both induce and sustain a Th1 immune response, and as described below, this response has been implicated in resistance against some helminths among them Taenia crassiceps infection $[34,35,36]$.

IL-12 signaling leads to activation of STAT4 by JAK2 and Tyk2 $[37,38]$. STAT4 dimers translocate into the nucleus and bind DNA sequences at the IFN- $\gamma$ activation site (GAS). In T cells and NK cells, the main action of IL-12 is the induction of IFN-Y [39]. By this pathway, STAT4 mediates most of the pro-inflammatory activities of IL-12 and is critical for Th1 differentiation and directing the cell-mediated immune response [40,41]. Rodriguez-Sosa and colleagues evaluated the role of STAT4 in experimental cysticercosis [34]. Surprisingly they found an unexpected participation of STAT4 in mediating resistance against this helminth. In this study, the authors observed that while wild type (WT) C57BL/ 6 mice were highly resistant to $T$. crassiceps infection, this group found that C57BL/6 mice lacking STAT4 (STAT4-/-) were highly susceptible to T. crassiceps infection. It is worth noting that in cysticercosis caused by $T$. crassiceps, there are sex-associated differences in susceptibility, where males are more resistant, and females show more susceptibility to this infection [42]. Although C57BL/ 6 is a resistant strain of mouse, these differences in susceptibility became evident in STAT4-/- mice, where both males and females became highly susceptible, but females still presented a greater parasite burden. In addition to the loss of resistance to this infection in STAT4 $\%$ mice, the Th1 immune response was also critically affected; they found a decrease in IgG2a specific antibodies, while there was an increase in IgG1 specific antibodies titers. The absence of STAT4 in this infection also affected splenocyte proliferation to specific antigens where they found an important decrease that was more evident as infection progressed. As expected, the absence of STAT4 decreased the levels of IFN- $\gamma$ from CD4+ enriched cells, whereas the levels of IL-13 and IL-10 were significantly increased.

Another case in which STAT4 appears to be involved is in the effect of vaccination on experimental schistosomiasis. Wynn et al [43] demonstrated that IL-12 enhances vaccine-induced immunity to schistosomes by augmenting both humoral and cell-mediated immune responses, and because IL-12 biological activity is STAT4-dependent, cysticercosis caused by T. crassiceps is not the only helminth infection related to STAT4 and protection. Interestingly, this protein has been also involved in anti-filarial responses. Those parasites are typical of tropical environments and are capable of avoiding immune response to facilitate their permanence in their host [44, 45]. Like other nematodes, filarias induce a Th2 response in their host [46]. Recent studies trying to un- 
cover how filarias avoid immune response have demonstrated that these parasites are capable of ontogenic transformations of surface epitope expression $[47,48]$. Besides surface antigens, Muthian and colleagues [35] demonstrated another immune evasion mechanism related to secreted lipids from the filarial parasite Setaria digitata, named, secreted filarial lipids (SFL). This group observed that proliferation of T cells induced by myelin antigens was decreased when co-stimulated with SFL, as well as IL-12 and IFN- $\gamma$ production. Given that IFN- $\gamma$ production is related to STAT4 activation, they analyzed the effect of SFL on STAT4 phosphorylation in IL-12-stimulated T cells; they observed that SFL decreased STAT4 activation in a dose-dependent manner. This same phenomenon was observed in the proteins responsible for STAT4 phosphorylation in response to IL-12, JAK2 and TyK2. Thus, the infection with $S$. digitata inhibits Th1 response by secreting lipid antigens that inhibit STAT4 activation favoring polarization toward a Th2 immune response. This suggests an important immune evasion mechanism where helminth-derived products drive host immune response toward a Th2 profile that, in some infections, works as an effector mechanism to remove parasites, but in other cases works as an evasion mechanism that favors establishment of the infection. Surprisingly, STAT4 has been recently implicated in Th2 immune response development; Syrbe et al [36] observed that although they employ Nippostrongylus brasiliensis, an infection model that triggers Th2 immune response, when mice lacking STAT4 were infected there was a decrease in the number of cells producing IL-4 . Thereby it is possible that STAT4 participates in Th2 immune response development, at least during $N$. brasiliensis infection.

\section{The Role of STAT6 in resistance to helminth infections}

It is generally recognized that IL-4 and IL-13-producing $\mathrm{CD}^{+}{ }^{+} \mathrm{Th} 2$ cells and eosinophils play an important role in inhibiting and killing helminth parasites and impeding helminth establishment and growth, as signature molecules associated with Th2-type immune responses are the predominant cytokines present either in circulation or at the site of infection during helminth parasitic infections [2]. These infections can be related to STAT6 because this transcription factor is a key molecule involved in IL-4 and IL-13 cytokine signaling [49]. The IL-4R and IL-13R share a common receptor chain involved in signal transduction. Both IL-4 and IL-13 are activators of STAT6, which mediates most biological activities of these cytokines. STAT6 $\%$ - mice display impaired Th2 differentiation and lose responsiveness to IL-4 and
IL-13, but these animals are capable to maintain normal responses to other cytokine signals. The importance of IL-4, IL-13 and STAT6 in mediating resistance during helminth infection was clearly demonstrated in experimental models of infection with the gastrointestinal parasite Trichinella spiralis. It has been observed that while IFN- $\gamma$ deficiency accelerates infection clearance, mice with IL-4R $\alpha$ deficiency did not achieve worm expulsion, nor did STAT6 $\%$ mice. The STAT6 $\%$ mice also displayed an increase in parasite burden and significant changes in cytokine profile production, evidenced by a decrease in IL-4 and IL-13 and increase in IFN- $\gamma$ production eleven days post infection. Moreover, it was observed that STAT6 was participating through mast cell-dependent mechanisms [50]. Thus, STAT6 and Th2 immune response are important in $T$. spiralis clearance.

Besides mast cell participation in T. spiralis expulsion, other important cell population that has been implicated in this process is the goblet cells, whose participation is STAT6-dependent, suggested by Khan and colleagues in 2001 [51]. This scientific group worked with STAT6 $/-$ mice and observed that these mice lost the resistance to infection comparing with WT mice, as demonstrated by higher parasite burden. Furthermore, it was also observed that the production of IL-4 and IL-13 was decreased 14 days post infection associated with a significant decrease in goblet cell numbers. In addition, the decrease in goblet cell production is important in the context that $T$. spiralis infection is characterized by an induced goblet cell hyperplasia necessary for worm expulsion $[52,53]$.

T. spiralis clearance has also been shown to be related to intestinal muscle hypercontractility. Mice strains that show a quick clearance of this worm also show greater gut muscle hypercontractility [54]. This hypercontractility depends on both $\mathrm{CD}^{+}$and MHC-II ${ }^{+} \mathrm{T}$ cells [55], although mechanisms involved in these events remain unclear. Thus, trying to determine possible mechanisms, in 2001 Khan and colleagues [56] analyzed the participation of IL-4 and STAT6 in hypercontractility development and its relationship with parasite expulsion. They observed that $T$. spiralis infection induced an increase in IL-4 and IL-6 cytokine production in mesenteric lymph node and spleen cell culture supernatants stimulated with ConA. However, this increase was not observed in STAT6 $\%$ mice. Together with these results, they found a marked attenuation of carbachol-induced muscle contraction in STAT6 $\%$ - mice but only a slight decrease in muscle hypercontractility in IL-4/- mice. Parasite burden was delayed in STAT6 $\%$ mice, but was almost normal in mice lacking IL-4. Thus, the 
absence of STAT6 is important in gut hypercontractility that can be related to the inefficiency of parasite expulsion. However, this seems to be an IL-4 independent event, so perhaps other cytokines such as IL-13 or IL-9 may be responsible for T. spiralis gastrointestinal infection clearance.

However, T. spiralis infection is not strictly restricted to the gastrointestinal site, but the larval stage of this parasite rapidly can get into the blood stream and disseminate in the host. In order to investigate the influence of Th2 cytokines during muscle infection with T. spiralis, Beiting et al [57] infected STAT6\% mice. These mice were in a BALB/c background, which would be expected to mount a strong Th2 response. Tissue muscle recovered from BALB/c mice at 18 dpi contained numerous mature larvae and fully developed nurse cells surrounded by focal infiltrates. In contrast, nurse cells from STAT6-/-infected mice had markedly reduced inflammatory infiltrates around infected cells and reduced numbers of lymph node cells. When stimulated with larval antigens, lymph node cells from BALB/c mice produced significantly more IL-4, IL-5, and IL-13, but less IFN- $\gamma$, compared with their STAT6\% counterparts, whereas IL-10 concentrations were comparable in BALB/c and STAT6 $\%$ mice. Interestingly, despite impairment of Th2 responses and inflammation in STAT6 $\%$ mice, larval burdens were similar to those in BALB/c mice. These data highlights a more important role for STAT6 in eliminating gastrointestinal worms than in tissue-dwelling helminths.

Another helminth parasite infection in which STAT6 appears to be involved is the gastrointestinal nematode parasite Nippostrongylus brasiliensis. The third-stage larvae of this parasite infect mice through the skin, migrate into the lungs, are coughed up, ingested, travel to gut lumen were mature adults reside, produce eggs that are excreted in the feces, and are themselves expelled approximately 10 days after the initiation of infection [58]. In 1988, Katona and colleagues [59] described that even when Th2 responses are necessary for parasite expulsion, IL-4 is not, while treatment of infected mice with IL-12 blocks worm expulsion [60]. Although those results suggest that the primary mechanism of host protection against $N$. brasiliensis might be production of a Th2 immune response, it seems to be IL-4-independent but may be related to other molecules involved in Th2 polarization, like IL-4 cytokine receptor IL-4R $\alpha$ or STAT6.

Urban and colleagues [4] analyzed whether $N$. brasiliensis expulsion was affected in mice lacking IL-4R $\alpha$ or STAT6. They found that both deficient animals failed to expel $N$. brasiliensis and presented elevated egg burdens, whereas WT mice did not.
These results were confirmed with the administration of anti-IL-4R $\alpha$ to WT mice, which lost resistance evidenced by the presence of egg and parasite burden. They also found antibody isotype changes, as WT mice produced IgG1 whereas IgG2a, characteristic in Th1 responses, was not detected. On the other hand, STAT $6 \%$ mice produced greater levels of both IgG1 and IgG2a antibodies during chronic infection. The fact that IL-4 is not the only cytokine that uses IL- $4 \mathrm{R} \alpha$ for signaling suggests analysis of the participation of IL-13, which also signals through IL-4R $\alpha$, might be appropriate. Administration of soluble IL-13R $\alpha 2-\mathrm{Fc}$, a fusion protein that neutralizes IL-13, in WT mice infected with $N$. brasiliensis, demonstrated that these mice became susceptible to infection. So, protection against $N$. brasiliensis depends mainly on STAT6 but not on IL-4, and STAT6 might be activated through IL-13 signaling, a cytokine related to Th2 immune response in addition to IL-4. This exhibits the importance of STAT6 in protection against gastrointestinal helminth infections.

Another enteric helminth related to this transcription factor is the cestode Hymenolepis diminuta. In 2003, McKay and Khan [61] described the importance of STAT6 in this infection by analyzing the participation of IL-4, IL-13 and STAT6 in worm expulsion. They found that adult worms were harbored in the gut only in STAT6\% mice, and that both IL-4 and IL-13 deficient mice were able to expel $H$. diminuta. Another important finding was the reduced goblet cell numbers found in STAT6 $/$ - mice that was related to susceptibility to infection.

Brugia malayi is a human-infecting tissue-dwelling nematode that induces a Th2 response that also seems to be beneficial in rodent hosts. In the mouse model of infection, immunocompetent mice are able to clear the parasites [62]. In 2001, Spencer and colleagues described that IL-4 is required for clearance of Brugia from BALB/c [63] and C57BL/6 mice strains. This was evidenced in IL-4 and STAT6 $\%$ mice, which still harbored live worms 6 weeks post-infection in BALB/c mice and two and four weeks post-infection in C57BL/6 mice, contrary to WT mice in both strains. Therefore, IL- 4 favors parasite clearance via the STAT6 signaling pathway in filariasis.

Another helminth infection is murine cysticercosis caused by the helminth parasite Taenia crassiceps, which induces a strong Th2-like response. Interestingly, T. crassiceps-infected mice develop a transient Th1-like response during the first weeks of infection, but later they develop a dominant Th2 response in chronic phases of infection ( $>4$ weeks) that is associated with an increase in parasite loads [64]. Trying to 
confirm that development of Th2 immune response correlates with susceptibility to this infection, Rodriguez-Sosa and colleagues [65] analyzed the role of a Th2-type response induced via STAT6-mediated signaling in BALB/c mice during T. crassiceps infection. They found that even when BALB/c mice were susceptible to infection and carried approximately 400 intraperitoneal metacestodes at 12 weeks after infection, STAT $6 \%$ mice resolved the infection as early as 4 weeks post-infection. STAT6 $/$ mice, together with resistance to infection, developed a dominant Th1-type response with high levels of anti-T. crassiceps-specific IgG2a antibodies, and lower levels of specific IgG1 and total IgE antibodies compared to WT mice; they also produced higher levels of IFN- $\gamma$ and lower levels of IL- 4 and IL-13 in the course of infection. Consistent with this, macrophages from STAT6 /- T. crassiceps-infected mice displayed an inflammatory phenotype with high IL-12 and NO production compared to macrophages from WT mice which displayed an alternative activation phenotype, with inhibited IL-12 production and NO and high expression of Arginase-1, PD-L2 and IL-4R $\alpha$ [66]. Together, these results show that murine cysticercosis requires a Th1 immune response development to clear infection, while Th2 immune response mediated by STAT6 is related to susceptibility to this extra-intestinal infection.

As previously described, while STAT6-mediated Th2 immune response is not always related to helminth resistance, it may be dependent on several factors, such as the site of infection, type of parasite and chronicity of the infection, among others. Thus, re- cently Mishra et al. [67] analyzed the development of immunopathology in a murine model of neurocysticercosis caused by Mesocestoides corti in the absence of STAT6. They found that WT BALB/c mice, as seen with $T$. crassiceps infection $[26,68,69,70]$, induced expression of markers associated with alternatively activated macrophages such as Ym1, Fizz1, Arg-1, MR1 and MGL $1 / 2$ in the brains of infected mice. Interestingly, STAT6-/- mice showed a down-regulation in the expression of AAMФ associated molecules compared with WT mice. As reported previously by Rodriguez-Sosa et al [71], STAT6 deficient mice with cysticercosis displayed also increased iNOS expression. However, these mice displayed enhanced susceptibility to $M$. corti and greater neuropathology (abnormal vestibular function, tilted head, walking in circles, and morbidity). Thus, it appears that in this model STAT6 may participate by controlling excessive pathology (inflammation) through the development of AAMФs, which have been proven to have suppressive activity mainly mediated by the Death-ligand pathway [66]. Furthermore, AАМФs may participate by healing central nervous system damage during neurocysticercosis, as one the newest functions associated with AАMФs during helminth infections [72]. Interestingly, the development of AAMФs and the expression of PD-L2 in such cells have been recently confirmed to be STAT6-dependent [73], suggesting that an anti-inflammatory and healing processes during helminth infections may be also associated with STAT6 function.

Table 3. STATs in helminth infections

\begin{tabular}{|c|c|c|c|}
\hline & Parasite & Effect & References \\
\hline STAT1 & Schistosoma mansoni & $\begin{array}{l}\text { Soluble egg antigens (SEA) do not induce STAT1 phosphorylation in macrophages but } \\
\text { induce ERK and p38 activation. }\end{array}$ & {$[30]$} \\
\hline \multirow[t]{3}{*}{ STAT4 } & Taenia crassiceps & Genetically resistant C57BL/6 mice became susceptible when lacking STAT4 gene. & {$[34]$} \\
\hline & Setaria digitata & $\begin{array}{l}\text { Secreted filarial lipids (SFL) down-regulate Th1 immune responses by decreasing } \\
\text { STAT4 phosphorylation in a dose-dependent manner. }\end{array}$ & {$[35]$} \\
\hline & Nippostrongylus brasiliensis & Decrease in frequency of IL-4 producing cells in infected STAT4-/-mice. & {$[36]$} \\
\hline \multirow[t]{8}{*}{ STAT6 } & Nippostrongylus brasiliensis & IL4-R $\alpha$ and STAT6 are necessaries for worm expulsion; IL-13 is decisive for clearance. & {$[4]$} \\
\hline & Trichinella spiralis & Absence of STAT6 is related to higher parasite burden and lower Th2 cytokines. & {$[50]$} \\
\hline & & STAT6 and Th2 cytokines seems to be necessary for goblet cell hyperplasia. & {$[51]$} \\
\hline & & $\begin{array}{l}\text { Gut muscle hyper-contractility necessary for worm expulsion depends on STAT6 } \\
\text { signaling. }\end{array}$ & {$[56]$} \\
\hline & Hymenolepis diminuta & $\begin{array}{l}\text { Resistance is mediated by STAT6 but do not depend on IL-4 or IL-13 alone. The ab- } \\
\text { sence of STAT6 down regulates goblet cells. }\end{array}$ & [61] \\
\hline & Brugia malayi & IL-4 and STAT6 are required for clearance from BALB/c and C57BL/6 mice. & {$[63]$} \\
\hline & Taenia crassiceps & STAT6 deficient mice were resistant to infection with an intense Th1 response. & {$[65]$} \\
\hline & Mesocestoides corti & $\begin{array}{l}\text { In murine neurocysticercosis STAT6 deficient mice succumbed faster given an intense } \\
\text { inflammatory response }\end{array}$ & {$[67]$} \\
\hline
\end{tabular}




\section{Conclusion}

The pathology and resolution of different helminth infections is dependent to a large extent on the infecting species, the model used, and the site of infection. STATs molecules do have a role in all cases either by initiating the development of a Th1 response as in schistosomiasis and cysticercosis or development of Th2 response in case of gastrointestinal infections. The major cytokines in both cases are IL-12, IFN-y, IL-10, IL-4, IL-13 the effects of which are mediated by specific STATs. Thus, even when STAT6 participates in the expulsion of some gut parasites, a STAT4 meditated Th1 immune response seems to be necessary to control extra-intestinal larvae stages. Hence, both of these STATs proteins are necessary in trigger immune responses against helminth infections but play different role. The fact that helminth-derived antigens have been demonstrated to inhibit at least STAT4 activation, it must be enough motivation to try new helminth antigens to study STAT modulation in different target cells, this will help to clarify how helminths modulate the immune response.

\section{Acknowledgments}

This work was supported by grants IN212909 PAPIIT-UNAM, PAPCA-FES Iztacala 23 (2010-2011), and ICyTDF. It is part of the requirements to obtain the PhD degree in the Postgraduate Program in Biomedical Sciences, Facultad de Medicina, UNAM, for M.B.D. who was supported by a fellowship from CONACYT-Mexico.

\section{Conflict of Interests}

The authors have declared that no conflict of interest exists.

\section{References}

1. Maizels R, Balic A, Gomez-Escobar N, Nair M, Taylor M, and Allen J. Helminth parasites-masters of regulation. Immunol Rev. 2004; 201: 89-116.

2. Pearce EJ, Reiner SL. Induction of Th2 responses in infectious diseases. Curr Opin Immunol. 1995 Aug;7(4):497-504.

3. King CL, Kumaraswami V, Poindexter RW, Kumari S, Jayara-man K, Alling DW, Ottesen EA, Nutman TB. Immunologic tol-erance in lymphatic filariasis. Diminished parasite-specific $\mathrm{T}$ and $\mathrm{B}$ lymphocyte precursor frequency in the microfilaremic state. J Clin Invest. 1992. May; 89(5): 1403-1410.

4. Urban JF Jr, Noben-Trauth N, Donaldson DD, Madden KB, Morris SC, Collins M, Finkelman FD. IL-13, IL-4Ralpha, and Stat6 are required for the expulsion of the gastrointestinal nematode parasite Nippostrongylus brasiliensis. Immunity. 1998 Feb;8(2):255-64.

5. Reyes J.L., Terrazas L.I. The divergent roles of alternatively activated macrophages in helminthic infections. Parasite Im-munol. 2007; 29: 609-619.
6. Gillan V, Devaney E. Regulatory $\mathrm{T}$ cells modulate Th2 responses induced by Brugia pahangi third-stage larvae. Infect Immun. 2005 Jul;73(7):4034-42.

7. Wilson MS, Mentink-Kane MM, Pesce JT, Ramalingam TR, Thompson R, Wynn TA. Immunopathology of schistosomiasis. Immunol Cell Biol. 2007;85(2):148-54.

8. Taylor MD, van der Werf N, Harris A, Graham AL, Bain O, Allen JE, Maizels RM. Early recruitment of natural CD4+ Foxp3+ Treg cells by infective larvae determines the zoutcome of filarial infection. Eur J Immunol. 2009 Jan;39(1):192-206.

9. Williams JG. STAT signalling in cell proliferation and in de-velopment. Curr Opin Genet Dev. 2000 Oct;10(5):503-7.

10. Darnell JEJ, Kerr IM, Stark GR. Jak-STAT pathways and tran-scriptional activation in response to IFNs and other extracellu-lar. Science. 1994;264(5164):1415-21.

11. Wilks AF, Harpur AG, Kurban RR, Ralph SJ, Zürcher G, Ziemiecki A. Two novel protein-tyrosine kinases, each with a second phosphotransferase-related catalytic domain, define a new class of protein kinase. Mol Cell Biol. 1991 Apr;11(4):2057-65.

12. Firmbach-Kraft I, Byers M, Shows T, Dalla-Favera R, Krolewski JJ. Tyk2, prototype of a novel class of non-receptor tyrosine ki-nase genes. Oncogene. 1990 Sep;5(9):1329-36.

13. Harpur AG, Andres AC, Ziemiecki A, Aston RR, Wilks AF. JAK2, a third member of the JAK family of protein tyrosine ki-nases. Oncogene. 1992. Jul;7(7):1347-53.

14. Johnston JA, Kawamura M, Kirken RA, Chen YQ, Blake TB, Shibuya K, Ortaldo JR, McVicar DW, O'Shea JJ. Phosphorylation and activation of the Jak-3 Janus kinase in response to in-terleukin-2. Nature 1994. 370:151-53.

15. Saharinen P, Takaluoma K, Silvennoinen O. Regulation of the Jak2 tyrosine kinase by its pseudokinase domain. Mol Cell Biol. 2000. May;20(10):3387-95.

16. Velazquez L, Fellous M, Stark G, and Pellegrini S. A Protein Tyrosine Kinase in the Interferon alpha/beta signaling Path-way. Cell. 1992 Jul 24;70(2):313-22.

17. Watling D, Guschin D, Muller M, Silvennoinen O, Witthuhn BA, Quelle FW, Rogers NC, Schindler C, Stark GR, Ihle JN, et al. Complementation by the protein tyrosine kinase JAK2 of a mutant cell line defective in the interferon-gamma signal transduction pathway. Nature. 1993. 366:166-70.

18. Silvennoinen $\mathrm{O}$, Ihle JN, Schlessinger J, Levy DE. Interfer-on-induced nuclear signalling by Jak protein tyrosine kinases. Nature 1993. 366:583-85.

19. Zhong Z, Wen Z, Darnell JE Jr. Science. Stat3: a STAT family member activated by tyrosine phosphorylation in response to epidermal growth factor and interleukin-6. Science. 1994 Apr 1;264(5155):95-8.

20. Hou J, Schindler U, Henzel WJ, Wong SC, McKnight SL. Iden-tification and purification of human Stat proteins activated in response to interleukin-2. Immunity. 1995;2(4):321-9.

21. Yamamoto K, Quelle FW, Thierfelder WE, Kreider BL, Gilbert DJ, Jenkins NA, Copeland NG, Silvennoinen O, Ihle JN. Stat4, a novel gamma interferon activation site-binding protein ex-pressed in early myeloid differentiation. Mol Cell Biol. 1994 Jul;14(7):4342-9.

22. Liu X, Robinson GW, Gouilleux F, Groner B, Hennighausen L. Cloning and expression of Stat 5 and an additional homologue $(S t a t 5 b)$ involved in prolactin signal transduction in mouse mammary tissue. Proc Natl Acad Sci U S A. 1995; 92(19):8831-5.

23. Quelle FW, Shimoda K, Thierfelder W, Fischer C, Kim A, Ruben SM, Cleveland JL, Pierce JH, Keegan AD, Nelms K, et al. Cloning of murine Stat 6 and human Stat6, Stat proteins that are tyrosine phosphorylated in responses to IL-4 and IL-3 but are not required for mitogenesis. Mol Cell Biol. 1995 Jun;15(6):3336-43.

24. Schindler CW. Series introduction. JAK-STAT signaling in human disease. J Clin Invest. 2002 May;109(9):1133-7. 
25. Rawlings JS, Rosler KM, Harrison DA. The JAK/STAT signaling pathway. J Cell Sci. 2004 Mar 15;117(Pt 8):1281-3.

26. Stein M, Keshav S, Harris N, Gordon S. Interleukin 4 potently enhances murine macrophage mannose receptor activity: a marker of alternative immunologic macrophage activation. J Exp Med. 1992 Jul 1;176(1):287-92.

27. Rodríguez-Sosa M, Rivera-Montoya I, Espinoza A, Romero-Grijalva M, López-Flores R, González J, Terrazas LI. Acute cysticercosis favours rapid and more severe lesions caused by Leishmania major and Leishmania mexicana infec-tion, a role for alternatively activated macrophages. Cell Immunol. 2006 Aug;242(2):61-71.

28. Zhu J, Yamane H, Paul WE. Differentiation of effector CD4 T cell populations. Annu Rev Immunol. 2010 Mar;28:445-89.

29. Pearce EJ. Priming of the immune response by schistosome eggs. Parasite Immunol 2005; 27:265-70.

30. Goh F, Irvine KM, Lovelace E, Donnelly S, Jones MK, Brion K, Hume DA, Kotze AC, Dalton JP, Ingham A, Sweet MJ. Selective induction of the Notch ligand Jagged-1 in macrophages by soluble egg antigen from Schistosoma mansoni involves ERK signalling. Immunology. 2009 Jul;127(3):326-37.

31. Wynn TA, Cheever AW, Jankovic D, Poindexter RW, Caspar P, Lewis FA, Sher A. An IL-12-based vaccination method for pre-venting fibrosis induced by schistosome infection. Nature. 1995 Aug 17;376(6541):594-6.

32. Mwatha JK, Kimani G, Kamau T, Mbugua GG, Ouma JH, Mumo J, Fulford AJ, Jones FM, Butterworth AE, Roberts MB, Dunne DW. High levels of TNF, soluble TNF receptors, soluble ICAM-1, and IFN-gamma, but low levels of IL-5, are associated with hepatosplenic disease in human schistosomiasis mansoni. J Immunol. 1998 Feb 15;160(4):1992-9.

33. Rosas LE, Keiser T, Pyles R, Durbin J, Satoskar AR. Development of protective immunity against cutaneous leishmaniasis is dependent on STAT1-mediated IFN signaling pathway. Eur J Immunol. 2003 Jul;33 (7):1799-805.

34. Rodríguez-Sosa M, Saavedra R, Tenorio EP, Rosas LE, Satoskar AR, Terrazas LI. A STAT4-dependent Th1 response is required for resistance to the helminth parasite Taenia crassiceps. Infect Immun. 2004. Aug;72(8):4552-60.

35. Muthian G, Pradeep C, Sargapradeep K, Kaleysaraj R, Bright J. Setaria digitata secreted filarial lipids modulate IL-12 signaling through JAK-STAT pathway leading to the development of Th1 response. Experimental Parasitology 2006. 114: 193-203.

36. Syrbe U, Hoffmann U, Schlawe K, Liesenfeld O, Erb K, Hamann A. Microenvironment-Dependent Requirement of STAT4 for the Induction of P-Selectin Ligands and Effector Cytokines on CD4+ T Cells in Healthy and Parasite-Infected Mice. The Journal of Immunology, 2006, 177: 7673-7679.

37. Schindler, C. and Darnell, J., Jr., Transcriptional responses to polypeptide ligands: the JAK-STAT pathway. Annu Rev Biochem.1995. 64: 621-651.

38. Ihle, J. N., Witthuhn, B. A., Quelle, F. W., Yamamoto, K. and Silvennoinen, O., Signaling through the hematopoietic cytokine receptors. Annu Rev Immunol. 1995. 13: 369-398.

39. Lund RJ, Chen Z, Scheinin J, Lahesmaa R. Early target genes of IL-12 and STAT4 signaling in th cells. J Immunol. 2004 Jun 1;172 (11):6775-82.

40. Jacobson NG, Szabo SJ, Weber-Nordt RM, Zhong Z, Schreiber RD, Darnell JE Jr, Murphy KM. Interleukin 12 signaling in $\mathrm{T}$ helper type 1 (Th1) cells involves tyrosine phosphorylation of signal transducer and activator of transcription (Stat) 3 and Stat4. J Exp Med. 1995 May 1;181(5):1755-62.

41. Kaplan MH, Sun YL, Hoey T, Grusby MJ. Impaired IL-12 re-sponses and enhanced development of Th2 cells in Stat4-deficient mice. Nature. 1996 Jul 11;382(6587):174-7.

42. Sciutto E, Fragoso G, Diaz ML, Valdez F, Montoya RM, Govezensky T, Lomeli C, Larralde C. Murine Taenia crassiceps cysticercosis: H-2 complex and sex influence on susceptibility. Parasitol Res. 1991;77(3):243-6.

43. Wynn TA, Reynolds A, James S, Cheever AW, Caspar P, Hieny S, Jankovic D, Strand M, Sher A. IL-12 enhances vac-cine-induced immunity to schistosomes by augmenting both humoral and cell-mediated immune responses against the par-asite. J Immunol. 1996 Nov 1;157(9):4068-78.

44. Maizels RM, Bundy DAP., Selkirk ME, Smith DF, Anderson RM. Immunological modulation and evasion by helminth par-asites in human populations. Nature 1993; 365: 797-805.

45. Maizel RM, Gomez-Escobar N, Gregory WF, Murray J, Zang X. Immune evasion genes from filarial nematodes. International Journal of Parasitology 2001; 31: 889-898.

46. Dalai SK, Das D, Kar SK. Setaria digitata adult 14- to 20-kDa antigens induce diferential Th1/Th2 cytokine responses in the lymphocytes of endemic normal and asymptomatic microfilar-iae carriers in bancroftian filariasis. Journal of Clinical Immu-nology 1998; 18: 114-123.

47. Bright JJ, Raj RK. Ontogenetic transformation of surface epitope expression, an adaptive immunoevasive strategy of filarial parasites. Indian Journal of Experimental Biology 1994; 32: 49-54.

48. Lawrence RA. Immunity to filarial nematodes. Veterinary Parasitology 2001. 100: 33-44.

49. Kaplan MH, Schindler U, Smiley ST, Grusby MJ. Stat6 is re-quired for mediating responses to IL-4 and for development of Th2 cells. Immunity. 1996 Mar;4(3):313-9.

50. Urban JF, Schopf L, Morris SC, Orekhova T, Madden K, Betts C, Gamble HR, Byrd C, Donaldson D, Else K, Finkelman FD. Stat6 signaling promotes protective Immunity against Trichinella spiralis through a mast cell- and T Cell-Dependent Mechanism. The Journal of Immunology, 2000, 164: 2046-2052.

51. Khan WI, Blennerhasset P, Ma C, Matthaei KI, Collins SM. Stat6 dependent goblet cell hyperplasia during intestinal nematode infection. Parasite Immunol. 2001 Jan;23(1):39-42.

52. Garside P, Grencis RK, McMowat A. T lymphocyte dependent enteropathy in murine Trichinella spiralis infection. Parasite Immunology 1992; 14: 217-225.

53. Finkelman FD, Wynn TA, Donaldson DD, Urban J.FJr. The role of IL-13 in helminth induced inflammation and protective immunity against nematode infections. Current Opinion in Immunology 1999; 11: 420-426.

54. Vallance BA, Blennerhassett PA, Collins SM. Increased intesti-nal muscle contractility and worm expulsion in nematode in-fected mice. Am. J. Physiol. 1997 35:G321-G327.

55. Vallance BA, Collins SM, Snider DP. CD4 T cells and major histocompatibility couple class II expression influence worm expulsion and increased intestinal muscle contraction during Trichinella spiralis infection. Infect Immun. 1999 67:6090-6097.

56. Khan WI, Vallance BA, Blennerhassett PA, Deng Y, Verdu EF, Matthaei KI, Collins SM. Critical role for signal transducer and activator of transcription factor 6 in mediating intestinal muscle hypercontractility and worm expulsion in Trichinella spi-ralis-infected mice. Infect Immun. $2001 \mathrm{Feb} ; 69(2): 838-44$.

57. Beiting DP, Gagliardo LF, Hesse M, Bliss SK, Meskill D, Ap-pleton JA. Coordinated control of immunity to muscle stage Trichinella spiralis by IL-10, regulatory T cells, and TGF-beta. J Immunol. 2007 Jan 15;178(2):1039-47.

58. Ogilvie BM, Hockley DJ. Effects of immunity on Nippostron-gylus brasiliensis adult worms: reversible and irreversible changes in infectivity, reproduction, and morphology. J Parasitol. 1968 54: 1073-1084.

59. Katona IM, Urban JFJr., Finkelman FD. The role of L3T41 and Lyt21 $\mathrm{T}$ cells in the $\operatorname{IgE}$ response and immunity to Nip-postrongylus brasiliensis. J Immunol. 1988; 140: 3206-3211.

60. Finkelman FD, Madden KB, Cheever AW, Katona IM, Morris SC, Gately MK, Hubbard BR, Gause WC, and Urban JFJr. Ef- 
fects of interleukin-12 on immune responses and host protection in mice infected with intestinal nematode parasites. J Exp. Med. 1994; 179: 1563-1572.

61. McKay DM, Khan WI. STAT-6 is an absolute requirement for murine rejection of Hymenolepis diminuta. J Parasitol. 2003 Feb;89(1):188-9.

62. Loke P, MacDonald AS, Allen JE. Antigen-presenting cells recruited by Brugia malayi induce Th2 differentiation of naïve CD4(+) T cells. Eur J Immunol. 2000 Apr;30(4):1127-35.

63. Babu S, Ganley LM, Klei TR, Shultz LD, Rajan TV. Role of gamma interferon and interleukin- 4 in host defense against the human filarial parasite Brugia malayi. Infect Immun. 2000 May;68(5):3034-5.

64. Terrazas LI, Bojalil R, Govezensky T, Larralde C. Shift from an early protective Th1-type immune response to a late permissive Th2-type response in murine cysticercosis (Taenia crassiceps). J Parasitol. 1998 Feb;84(1):74-81.

65. Rodríguez-Sosa M, David JR, Bojalil R, Satoskar AR, Terrazas LI. Cutting edge: susceptibility to the larval stage of the helminth parasite Taenia crassiceps is mediated by Th2 response induced via STAT6 signaling. J Immunol. 2002 Apr 1;168(7):3135-9.

66. Terrazas LI, Montero D, Terrazas CA, Reyes JL, Rodríguez-Sosa $\mathrm{M}$. Role of the programmed Death-1 pathway in the suppressive activity of alternatively activated macrophages in experimental cysticercosis. Int J Parasitol. 2005 Nov;35(13):1349-58.

67. Mishra BB, Gundra UM, Teale JM. STAT6-/- mice exhibit de-creased cells with alternatively activated macrophage pheno-types and enhanced disease severity in murine neurocysticer-cosis. J Neuroimmunol. 2011 Mar;232(1-2):26-34.

68. Munder M, Eichmann K, Modolell M. Alternative metabolic states in murine macrophages reflected by the nitric oxide synthase/arginase balance: competitive regulation by CD4+ T cells correlates with Th1/Th2 phenotype. J Immunol. 1998 Jun 1;160(11):5347-54.

69. Raes G, De Baetselier P, Noël W, Beschin A, Brombacher F, Hassanzadeh Gh G. Differential expression of FIZZ1 and Ym1 in alternatively versus classically activated macrophages. J Leukoc Biol. 2002 Apr;71(4):597-602.

70. Raes G, Brys L, Dahal BK, Brandt J, Grooten J, Brombacher F, Vanham G, Noël W, Bogaert P, Boonefaes T, Kindt A, Van den Bergh R, Leenen PJ, De Baetselier P, Ghassabeh GH. Macro-phage galactose-type C-type lectins as novel markers for alter-natively activated macrophages elicited by parasitic infections and allergic airway inflammation. J Leukoc Biol. 2005 Mar;77 (3):321-7.

71. Rodríguez-Sosa M, Satoskar AR, Calderón R, Gomez-Garcia L, Saavedra R, Bojalil R, Terrazas LI. Chronic helminth infection induces alternatively activated macrophages expressing high levels of CCR5 with low interleukin-12 production and Th2-biasing ability. Infect Immun. 2002 Jul;70(7):3656-64.

72. Allen JE, Maizels RM. Diversity and dialogue in immunity to helminths. Nat Rev Immunol. 2011 Jun; 11(6):375-88.

73. Huber S, Hoffmann R, Muskens F, Voehringer D. Alternatively activated macrophages inhibit T-cell proliferation by Stat6-dependent expression of PD-L2. Blood. 2010 Oct 28;116(17):3311-20. 